\title{
Fluid detection with photonic crystal-based multichannel waveguides
}

\author{
J. Topol'ančik, P. Bhattacharya, ${ }^{\text {a) }}$ J. Sabarinathan, and P.-C. Yu \\ Solid State Electronics Laboratory, Department of Electrical Engineering and Computer Science, \\ University of Michigan, Ann Arbor, Michigan 48109-2122
}

(Received 22 August 2002; accepted 30 December 2002)

\begin{abstract}
A simple fluid detection scheme, based on light propagation through linear defect waveguides in photonic crystals, is demonstrated with isopropanol and xylene. The two-channel photonic crystal waveguide sensor is made from a GaAs-based heterostructure. The preferential channeling of light is controlled by the change in the refractive index of the corresponding waveguide branch due to the presence of the inserted fluid in the guide regions only. () 2003 American Institute of Physics.
\end{abstract}

[DOI: $10.1063 / 1.1554772$ ]

Optical waveguides based on photonic crystal (PC) structures ${ }^{1}$ offer a means of achieving strong optical confinement and low-loss waveguiding through optical circuits with sharp bends on the order of wavelength of the guided light. $^{2-13}$ In this letter, we demonstrate that PC slab waveguides can be used to design microfluidic sensors and can serve as building blocks for micron-scale fluid detection system. The operation principle of these devices is based on the change in light transmission through a waveguide branch induced by change of the refractive index of a defect with the fluid. PC's of air columns in a high refractive index slab appear to be ideal candidates for realizing this detection scheme as they allow fluids to penetrate the air columns and change the transmission through linear defect guides.

Our detection scheme was originally designed to distinguish between isopropanol $(n=1.3776)$ and xylene $(n$ $=1.5002$ ). As shown in Fig. 1, a single branched waveguide structure in a triangular photonic lattice is employed. The angular geometry is chosen to eliminate a direct beam path between light that is not coupled to the waveguide and the detector. The input waveguide has a row of missing holes as defects, while the two fluid detection branches consist of waveguides with holes smaller than those in the PC bulk $(r / a=0.37)$. For our design $a=0.56 \mu \mathrm{m}$ and $r / a=0.26$ and 0.32 for the two waveguide branches. The optimal PC-slab thickness of $d=0.6 a(\approx 0.34 \mu \mathrm{m})$ is chosen, ${ }^{14}$ and the effective refractive index approximation is used to determine the index of the fundamental propagation mode. This index value is used in two-dimensional frequency domain planewave expansion technique that is employed to obtain the band structure. ${ }^{15}$ The calculated dispersion relations for the guided transverse electric (TE)-like modes (without the presence of any fluid, i.e., with only air) corresponding to the three kinds of defects are shown in Fig. 2(a). Our excitation frequency is chosen at $a / \lambda=0.358$ within the range $(0.356-$ 0.361 ) of the input channel mode (defect 0 ) which couples to the missing defect (defect 0) mode, but not to the defect modes of the two detection channels (defect mode 1 and 2). If only the holes of the detection channels are now filled with fluids of different refractive indices, the frequencies of the guided modes are lowered, as shown in Figs. 2(b) and 2(c).

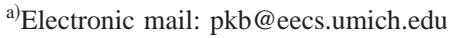

In our particular design when isopropanol is present, defect 2 mode couples to the excitation frequency but not defect 1 mode and vice versa when xylene is present. Therefore, for a fixed input excitation, the guides can be designed such that only one of the two branches will transmit light with a particular fluid.

The branched waveguide detector was fabricated with a molecular-beam epitaxially grown heterostructure consisting of a $0.34 \mu \mathrm{m}$ thick GaAs waveguide layer with a $1.5 \mu \mathrm{m}$ thick $\mathrm{Al}_{0.9} \mathrm{Ga}_{0.1} \mathrm{As}$ sacrificial layer below it, on a GaAs substrate. The photonic crystal consisting of air holes (Fig. 3) was fabricated with the help of electron-beam lithography and $\mathrm{Cl}_{2}$-based reactive ion etching ( $\mathrm{RIE}$ ), with silicon nitride serving as an etch mask. Scanning electron microscope (SEM) images indicate that our fabricated defect dimensions are within $5 \%$ of the design parameters. An air bridge is formed by a selective $\mathrm{HF}$ etch that removes the sacrificial $\mathrm{Al}_{0.9} \mathrm{Ga}_{0.1}$ As layer through the PC air holes. To cover the bulk PC air holes, a $0.4 \mu \mathrm{m}$ thick layer of silicon dioxide $\left(\mathrm{SiO}_{x}\right)$ is deposited at a steep angle $\left(\sim 80^{\circ}\right)$ and another electron-beam lithography step, followed by $\mathrm{CHF}_{3}$-based silicon oxide RIE etch, were performed to expose the defect guides only. The thick $\mathrm{SiO}_{x}$ layer covering the bulk of the sample enhanced the structural stability and ensured that the fluids to be tested did not fill the holes in the bulk PC. This oxide layer is taken into account in the calculation of the dispersion calculations. It may be noted that the angular

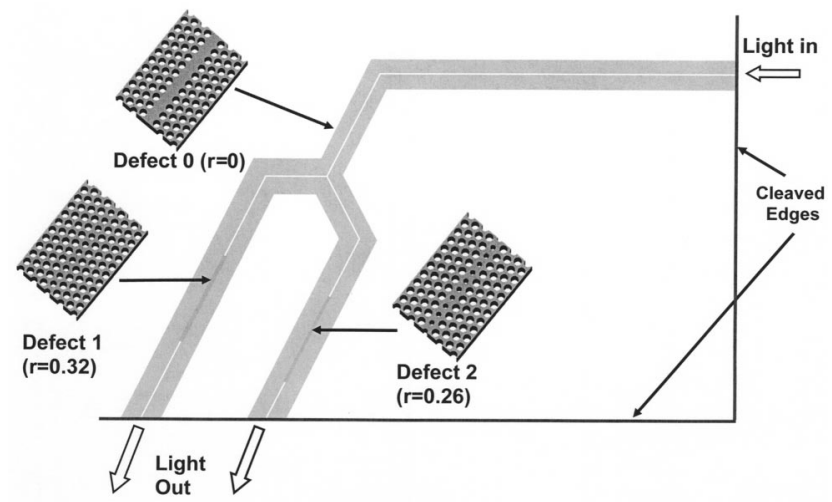

FIG. 1. Schematic representation of the PC branched waveguide microfluidic detector. 


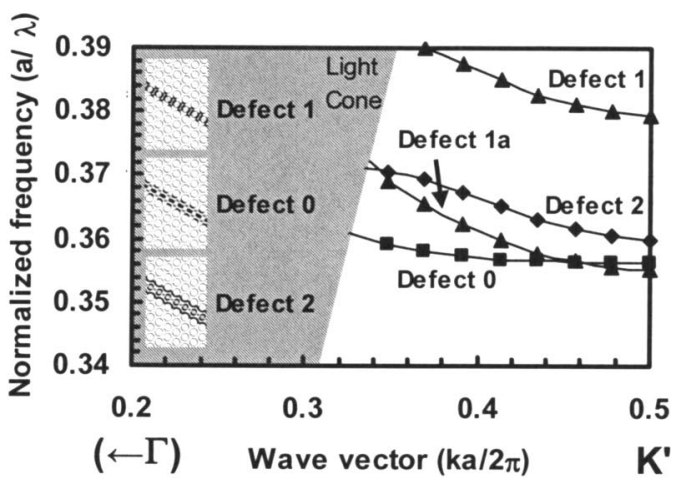

(a)

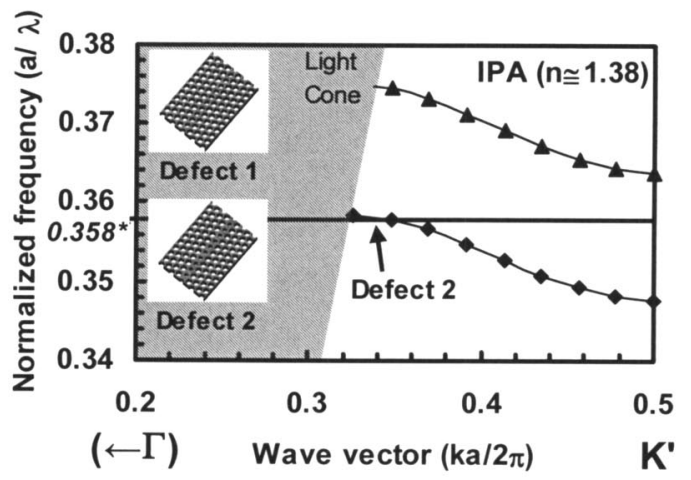

(b)

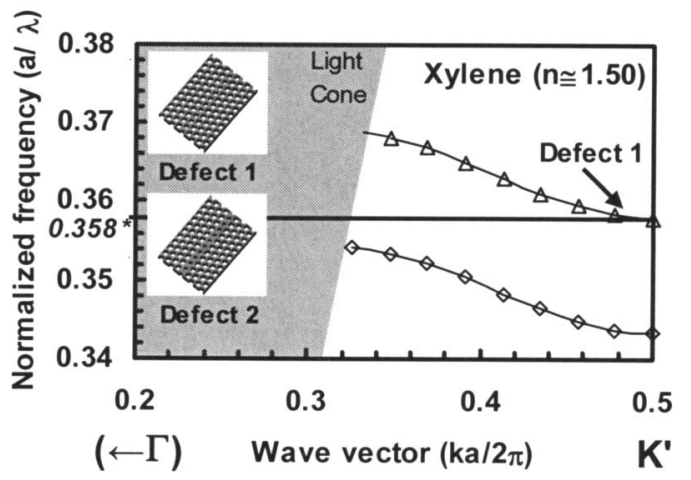

(c)

FIG. 2. (a) Dispersion diagram showing normalized frequency versus inplane wave vector for three defect TE-like modes. Defect 0 is a row of missing holes and defects 1 and 2 have a row of holes with $r / a=0.32$ and 0.26 , respectively. Note that defect 1 has an additional mode (defect 1a) at the excitation frequency which is uncoupled with the addition of either fluid. The insets depict the time-averaged electric field densities in the three waveguides, (b) the effect of isopropyl alcohol $(n=1.38)$ on both defect modes showing only defect 2 mode coupled, and (c) the effect of xylene (1.50) showing only defect 1 mode coupled at the excitation frequency $a / \lambda=0.358$.

deposition of $\mathrm{SiO}_{x}$ ensures that the $\mathrm{PC}$ air holes are not filled.

A color center laser, tunable in the wavelength range from 1500 to $1620 \mathrm{~nm}$, was used as the optical source. The laser output was coupled directly to the cleaved edge of the waveguide using an objective lens with a low focal ratio $(f / D \approx 2)$, that focused the $50 \mathrm{~mW}$ beam into a spot of estimated size 3-4 $\mu \mathrm{m}$. The guided output at the cleaved waveguide facet was observed with an infrared camera placed in the plane of the waveguide. The spectral transmis-

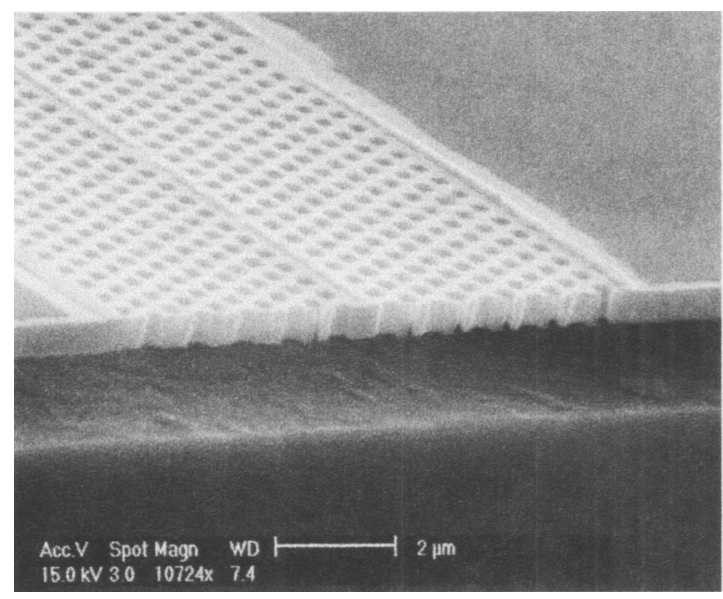

FIG. 3. SEM image of fabricated PC defect waveguide structure before $\mathrm{SiO}_{x}$ deposition.

sion characteristics of a defect waveguide section with a $60^{\circ}$ bend, as shown in the inset of Fig. 4(a), were first measured. This guide has the same design characteristics (missing holes) as the input guide of the dual-channel microfluidic detector. The excitation beam was coupled to the waveguide directly with the focusing lens and the transmitted light was collected with a single-mode fiber connected to an HP 7095B Optical Spectrum Analyzer. As seen in Fig. 4(a), an increase in transmitted intensity is observed around $1600 \mathrm{~nm}$, which is $\sim 30 \mathrm{~nm}$ higher than the value predicted by the band structure calculation. The difference can be attributed to fabrication errors and the validity of the effective refractive index approximation, which is discussed by Painter et al. ${ }^{16}$

To test the efficiency of fluid detection with the PC sensor, the wavelength of the input excitation was adjusted such that the light transmitted through both defect waveguide branches was minimized. In terms of the defect mode structure, this corresponds to a frequency below the defect modes in both branches. The transmitted light is detected with a $\mathrm{PbO}-\mathrm{PbS}$-Vidicon infrared camera and observed on a monitor. With the transmission set at a minimum, a small amount of isopropanol was introduced with a syringe onto the surface of the detector. Care was taken to ensure that the fluid did not flow over the cleaved edges. While the transmitted intensity of both channels increased, the intensity increased much more significantly at the output of the channel having a defect guide fabricated with $r / a=0.24$, for the detection of isopropanol. The effect is attributed to the downward shift of the defect mode in frequency, as predicted by the dispersion relations. The images of the transmitted light on the monitor, without any fluid and with isopropanol are shown in Figs. 4(b) and 4(c), respectively. Here we need to point out that the fabricated $r / a=0.24$, based on a design without taking the oxide into consideration, differs from the design $r / a=0.26$ for the defect 2 channel and the effect of this discrepancy is explained later. The observed change disappears in a few seconds as the fluid evaporates. Similarly, when xylene was inserted into the defect waveguides, a relatively larger increase in transmission was observed for the other channel, having the defect guide with $r / a=0.32$.

It was observed that the guiding in both channels could not be eliminated altogether when no fluid was present as predicted by the design. This was later found to be due to the 


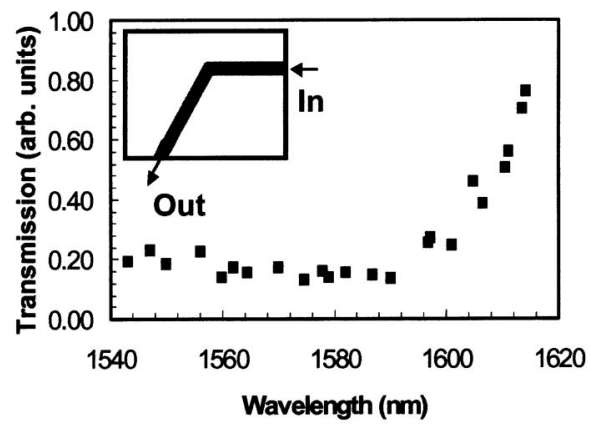

(a)

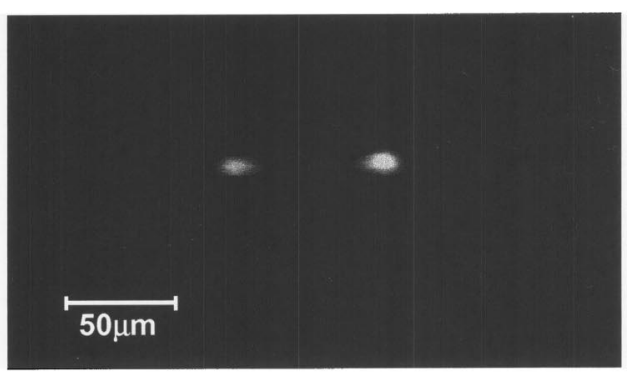

(b)

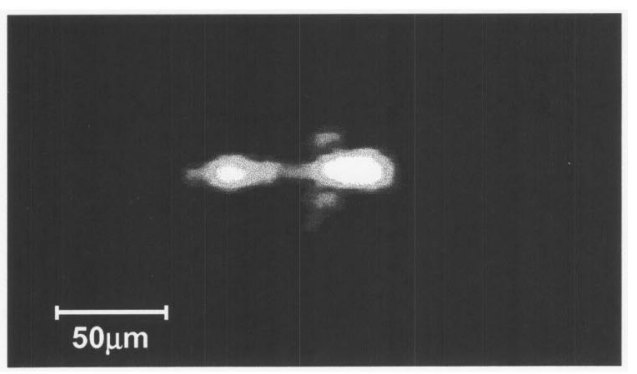

(c)

FIG. 4. (a) Measured transmission spectrum of a defect waveguide with a row of missing holes. The waveguide configuration is shown in the inset; (b) image of transmitted output in two-channel branched detector without fluid; and (c) image of transmitted output with isopropyl alcohol in the waveguide regions.

presence of a defect 1a mode at the excitation frequency as shown in Fig. 2(a). This defect 1a mode is an antisymmetric mode that couples very weakly to the symmetric feeding mode (defect 0 ), which explains significantly lower transmission intensity [Fig. 4(b)] than that of the symmetric defect modes activated by fluids [Fig. 4(c)]. This mode is also uncoupled in the presence of either fluid. This feature can be eliminated in future designs by replacing the whole missing defect input channel with a smaller defect hole channel. The design also predicts that while the transmission through one channel increases, the transmission through the other should be zero. It is evident that we were only able to observe relative changes in light transmission due to fluid insertion, rather than transmission completely through one channel alone for each fluid. This can be attributed to discrepancies between the calculated design values and actual fabricated values. As these devices have a very low tolerances, this variation probably causes just enough overlap of the two defect modes and thus reduces the resolution of the switch in light intensities between the channels with the different fluids. Modifications can be made to this basic design to improve the tolerances of these devices and extend these devices to multiple channels and fluids. This can be done by replacing the missing defect input waveguide (defect 0 mode) with smaller hole defect guides thus increasing our range of operation. The second method is to increase the width of the channels to increase the number of the modes to manipulate and thereby increase the number of fluids to be detected. Finally one should note that leaky modes are not observed at the output of these devices. In test measurements on such PC waveguides, where most of the light coupled to leaky modes, we observed negligible light output. After three to four bends, losses from the defect region holes and significant loss over the length of the waveguide traversed, the leaky modes do not make it to the output of the waveguides. The light observed from these devices is that of the photonic crystal guided defect modes.

This work is supported by the Defense Advanced Research Projects Agency under Grant No. MDA972-00-10020 and the Army Research Office under Grant No. DAAD19-01-1-0527. The work was performed, in part, at the Cornell Nanofabrication Facility, which is supported by the National Science Foundation under Grant No. ECS9731293.

${ }^{1}$ E. Yablonovitch, Phys. Rev. Lett. 58, 2059 (1987).

${ }^{2}$ A. Mekis, J. C. Chen, I. Kurland, S. Fan, P. R. Villeneuve, and J. D. Joannopoulos, Phys. Rev. Lett. 77, 3787 (1996).

${ }^{3}$ S.-Y. Lin, E. Chow, V. Hietala, P. R. Villeneuve, and J. D. Joannopoulos, Science 282, 274 (1998).

${ }^{4}$ N. Kawai, K. Inoue, N. Carlsson, N. Ikeda, Y. Sugimoto, K. Asakawa, and T. Takemori, Phys. Rev. Lett. 86, 2289 (2001).

${ }^{5}$ P. R. Villeneuve, S. Fan, S. G. Johnson, and J. D. Joannopoulos, IEE Proc.-J: Optoelectron. 145, 384 (1998).

${ }^{6}$ M. Lončar, T. Doll, J. Vučković, and A. Scherer, J. Lightwave Technol. 18, $1402(2000)$

${ }^{7}$ M. Kanskar, P. Paddon, V. Pacradouni, R. Morin, A. Busch, J. F. Young, S. R. Johnson, J. MacKenzie, and T. Tiedje, Appl. Phys. Lett. 70, 1438 (1997).

${ }^{8}$ Y. Sugimoto, N. Ikeda, N. Carlsson, K. Asakawa, N. Kawai, and K. Inoue, J. Appl. Phys. 91, 922 (2002).

${ }^{9}$ T. Baba, N. Fukaya, and J. Yonekura, Electron. Lett. 35, 654 (1999).

${ }^{10}$ A. Talneau, L. Le Gouezigou, N. Bouadma, M. Kafesaki, C. M. Soukoulis, and M. Agio, Appl. Phys. Lett. 80, 547 (2002).

${ }^{11}$ M. Lončar, D. Nedeljković, T. Doll, J. Vučković, A. Scherer, and T. P. Pearsall, Appl. Phys. Lett. 77, 1937 (2000).

${ }^{12}$ S. Yamada, T. Koyama, Y. Katayama, N. Ikeda, Y. Sugimoto, K. Asakawa, N. Kawai, and K. Inoue, J. Appl. Phys. 89, 855 (2001).

${ }^{13}$ Y. Sugimoto, N. Ikeda, N. Carlsson, K. Asakawa, N. Kawai, and K. Inoue, Appl. Phys. Lett. 79, 4286 (2001).

${ }^{14}$ S. Johnson, P. Villeneuve, S. Fan, and J. D. Joannopoulos, Phys. Rev. B 62, $8212(2000)$.

${ }^{15}$ R. D. Meade, A. M. Rappe, K. D. Brommer, and J. D. Joannopoulos, Phys. Rev. B 48, 8434 (1993).

${ }^{16}$ O. Painter, J. Vučković, and A. Scherer, J. Opt. Soc. Am. B 16, 275 (1999). 